the ganglioneuroma is sugically removed. The child was discharged with low weight heparine.

Conclusion Von Recklinghausen disease generally has a good prognosis. Major risks for morbidity and mortality are vascular complications. In case of pulmonary hypertension in these patients early diagnosis and sufficient therapy is essential to avoid major complications. Pulmonary embolism and NF as a cause for pulmonary hypertension has not been described before.

\section{MALIGNANT PERTUSSIS IN THE YOUNG INFANT: A CASE REPORT}

doi:10.1136/archdischild-2012-302724.0571

M Benedit, I Llana, MT Garcia de Alvaro, M Fernandez, S Jimeno. Hospital HM Torrelodones, Madrid, Spain

Infections by bordetella pertussis, has resurged in many countries around the world including developed regions. Where as new vaccination strategies for adolescents, pregnant women and adults have been recommended, mortality afecting young infants is still significant. Patients with severe cases present with extreme leukocytosis and develop refractory hypoxemia and pulmonary hypertension that is unresponsive to maximal intensive care.

Objective To report a case of malignant pertussis in a 4 weeks old infant.

Design a descriptive case report

Patient a 4 weeks old boy was admitted to the intensive Care Unit with respiratory distress and a diagnosis of bronchiolitis. Both parents and a 19 months old sibbling had upper respiratory infection symptoms. Four days before admission he started with coryza and cough. The cough was not paroxismal and cianosis and whooping were never present. He presented a marked leucocytosis wih lymphocytosis. Respiratory failure occurred rapidly from the fourth day with subsequent deterioration due to the development of severe pulmonary hypertension and multiorganic failure which caused the infant's death after 4 days of intensive care therapy.

Although Bordetella Pertussis was not confirmed by PCR, culture o serology the findings on the autopsy were suggestive of malignant pertussis.

Comments mortality due to malignant pertussis remains superior to $75 \%$ regardless life suport measures. Adults are the main source of contamination of non-immunized infants, thus immunization of older adolescents and adults who will have close contact with infants aged $<12$ months is the most effective preventive measure.

\section{INTRACTABLE DIARRHEA FROM CYTOMEGALOVIRUS COLITIS IN AN IMMUNOCOMPETENT ADOLESCENT}

doi:10.1136/archdischild-2012-302724.0572

${ }^{1} \mathrm{O}$ Canan, ${ }^{2} \mathrm{~S}$ Asilsoy, ${ }^{3} \mathrm{~N}$ Bal, ${ }^{4} \mathrm{M}$ Özkale, ${ }^{5} \mathrm{~A}$ Noyan, ${ }^{1} \mathrm{~F}$ Ozcay. 'Pediatric Gastroenterology; ${ }^{2}$ Pediatric Allergy; ${ }^{3}$ Pathology; ${ }^{4}$ Pediatric; ${ }^{5}$ Pediatric Nephrology, Baskent University, Adana, Turkey

Introduction We reported CMV colitis in an immunocompetent adolescent who was critically ill with septicaemia and significant intractable diarrhea that responded to specific CMV treatment.

Case A 15-year-old boy who previously known hereditary spherocytosis was referred to our hospital because of anemia, increased transaminase level, and massive cholestasis. The abdominal ultrasonography was detected cholelithiasis, choledoch stones, and dilated proximally bile ducts. External biliary drainage tube was placed into the choledoch. On the following days, spleenic rupture and sepsis developed. Therefore, the patient was underwent splenectomy and cholecystectomy. After closed of external biliary drainage tube, patient was developed severe dehydration and malnutrition due to watery stool. The colonoscopy and colonic biopsies was performed. It was shown macroscopic colitis and CMV intranuclear inclusion bodies in rectosigmoid colon. Morever, PCR for CMV DNA in blood $(6142 \mathrm{copy} / \mathrm{mL}$ ) and colonic biopsy speciments was positive. The immunologic screen tests were normal. Parenteral gancyclovir for 21 days and oral gancyclovir therapy was continued two weeks. The patient resolved completely, serum PCR for CMV DNA was detected negative after two months.

Conclusion CMV colitis, although rare in immunocompetent adolescent, should be considered in the differential diagnosis of severe colitis when other causes fail to explain the course of disease.

\section{UNCOMMON PRESENTATION OF UMBILICAL SEPSIS IN NEWBORN CAUSED BY HERPES INFECTION}

doi:10.1136/archdischild-2012-302724.0573

A Silwal, V Kumar. Department of Paediatrics, Calderdale and Huddersfield NHS Trust, Halifax, UK

Herpes simplex virus (HSV) is a widespread pathogen. Comparatively neonatal HSV infections are rare but frequently severe with high mortality and morbidity. Early detection and treatment, has been shown to decrease the mortality significantly.

We present a rare presentation of neonatal herpes in an 8 day old neonate who presented with fever and pustules around the umbilicus. Baby was treated with intravenous benzylpenicillin and flucloxacillin. However, baby was still febrile after 48 hours of treatment with vesicular lesions now appearing around the umbilicus. There was no history of genital herpes or cold sores in family. Viral swab was taken and acyclovir started. Wound swab and blood PCR was positive for HSV 2. Lumbar puncture done at day 14 was clear. Baby was treated with two weeks of intravenous acyclovir. There were problems with intravenous access with difficult access and later central line causing thrombosis.

Baby represented at 5 weeks age with vesicular umbilical lesions and was treated with 2 weeks course of oral acyclovir.

Many issues were highlighted in this case.

Lumbar puncture was not done early on as umbilical sepsis was thought to be focus of infection. It was difficult to establish whether this neonate had CNS herpes.

Long term intravenous treatment is easily said than can be given. This neonate had difficult access; central line was not without complication. What is the best way forward?

The lesions recurred within 2 weeks of the baby going home. Suppressive Acyclovir therapy - Would it be beneficial for him?

\section{VARICELLA ZOSTER INFECTION COMPLICATED BY MENINGEOCOCCALMENINGITS, SEOSIS AND SUBDURAL EMPYEMA IN AN INFANT, A CASE REPORT}

doi:10.1136/archdischild-2012-302724.0574

T Hassan, B Jamal, I Ahmed. Paediatrics, University Hospital of Limerick, Limerick, Ireland

Aims To highlight possible and may be fatal complications of Varicella Zoster virus (VZV) infection.

Method We report a case of an infant with VZV infection who developed meningeococcal sepsi, meningeococcal meningitis, VZV encephalitis and subdural empyema.

Results A 3 month, previously well and developmentally normal male child was admitted with skin rash. He had contact with varicella zoster infection. His initial inflammatory markers were high. He was treated as Chicken pox. His blood culture grew Nisseria Meningitidis, treated with intravenous antibiotics. He remained unwell with poor response to antibiotics. A lumbar puncture was then performed. This revealed a picture consistent with bacterial meningitis. CSF PCR was positive for $\mathrm{N}$. Meningitidis as well as for VZV.A week after admission he started to have seizures. This required intubation and PICU admission. A brain CT showed to 
bilateral subdural collection. He was assessed at 10 month of age and found to have no residual disabilities.

Conclusion VZV infection complicated by meningococcal sepsis and meningitis is well reported in the literature; subdural empyema is reported on few occasions; however we found no report with all these complications in a single patient nor in an infant.

\section{ILIOPSOAS ABSCESS IN THE NEONATE WITH IMMUNODEFICIENCY}

doi:10.1136/archdischild-2012-302724.0575

N Karabayir, 0 Turel, C Aydogmus, N Hatipoglu, A Hocaoglu, E Adal. Kanuni Sultan Suleyman Training and Research Hospital, Istanbul, Turkey

Psoas abscess is rare, especially in the neonatal period. It may be primary or rarely secondary to extend on from adjacent structures or to bacteremia caused by distant cutaneous infections. Vague clinical presentation may lead to delayed diagnosis. Appropriate drainage is necessary in addition to antibiotic therapy. Here we present a neonate was admitted with subcutaneous abscesses on his wrist and ankle. Staphylococcus aureus was isolated from the drainage material and proper antibiotic treatment was begun. On the $7^{\text {th }}$ day of treatment, he developed swelling on his groin and limited hip motion. Septic arthritis was suspected and a magnetic resonance imaging performed revealing an abscess on the right psoas muscle. Drainage and antibiotic treatment led to resolution of abscess. Development of multiple subcutaneous and deep abscesses in newborn period led us to suspect of primary immunodeficiency. In the immunological work up, serum immunoglobulins and lymphocyte sub set analysis were in normal ranges according to age. The phagocytic cell functions were tested with nitroblue-tetrazolium (NBT) slide test were also normal. Flow cytometry analyses revealed CD18 $16 \%, \mathrm{CD} 11 \mathrm{a}+\mathrm{CD} 18$ 17\%, CD11b + CD18 3\%, CD11c + CD18 4\%. A ratio of 1.89 oxidative explosion, 1.63 phagocytosis and 1.43 chemotaxis $(\mathrm{N}$ : $>1.5)$ was determined in neutrophil function tests. Antibiotic prophylaxis was initiated and bone marrow transplantation was recommended. As a conclusion, immunodeficiency syndromes such as LAD should be investigated in newborn with psoas abscess. Our case is important to emphasize underlying factors in the pathogenesis of psoas abscess.

\section{GENITAL NECROTISING FASCITIS IN A PREMATURE NEONATE SECONDARY TO GROUP B STREPTOCOCCUS(GBS) SEPSIS}

doi:10.1136/archdischild-2012-302724.0576

SA Ahmed, P Sivakumar. Neonatology, Luton \& Dunstable Hospital NHS Foundation Trust, Luton, UK

Background Necrotising Fascitis (NF) is primarily an adult disease but there are pediatric case series also. In the neonate, most cases of NF are attributable to secondary infection of omphalitis, balanitis, mammitis, postoperative complications, and fetal monitoring Other associations of NF included necrotizing enterocolitis, immunodeficiency and septicemia.

Method Case report and literature review.

Results Baby boy $\mathrm{S}$ is a 33 wks gestation with birth weight of 1.9 kgs born to a 21 yr old mum with uneventful pregnancy. She did not have high vaginal swab screening for GBS during pregnancy. He was born in good condition not needed resuscitation. He was cardiorespiratory stable on nasogastric feeds until day 5 when he developed grunting and tachypnoea requiring intubation and ventilation. $\mathrm{He}$ required both conventional \& High Frequency Oscillatory Ventilation. He was extubated to CPAP on day 13

He grew Group B Streptococcus (GBS) on blood and CSF culture. He was treated with a 3 week course of IV cefotaxime \& benzylpenicillin and was commenced on oral pencillin prophylaxis for 3 months. Localised scrotal skin breakdown noted on day 11 with a rapidly progressive inflammation, necrosis and gangrene skin subcutaneous tissues. Regular dressing with duoderm, supportive care and I.V antibiotic has resolved necrotising fascitis with residual scar

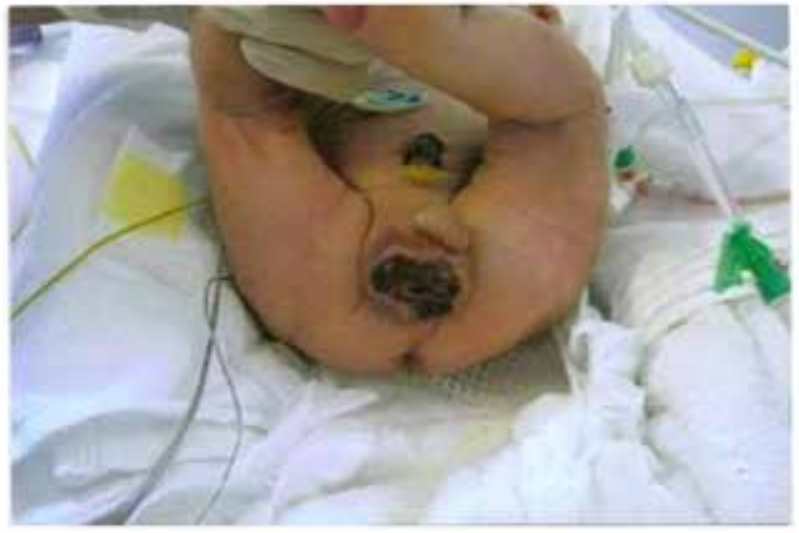

Genital Necrotising fascitis

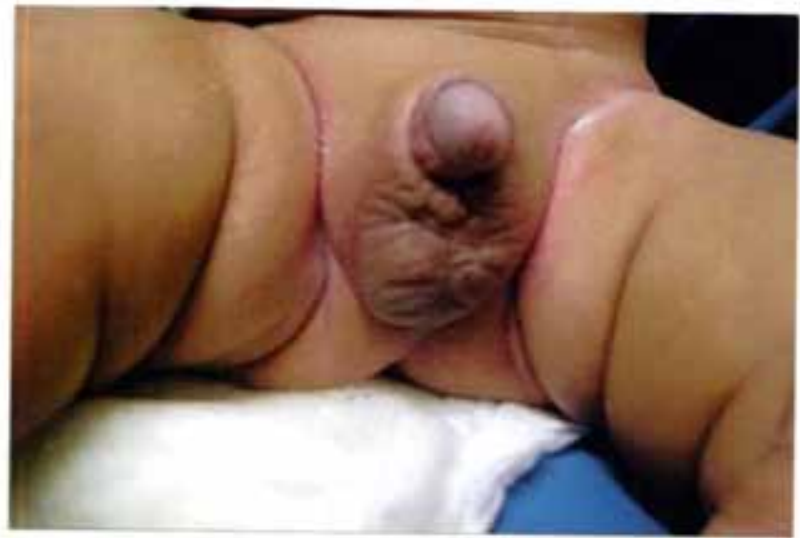

Genital llecrotising fascitis post treatment

\section{Abstract 576 Figure 1}

Conclusion We report the first case in literature of Genital Necrotising Fascitis in premature baby with Group B Streptococcus sepsis and meninigitis. It is relatively rare and has a fulminant course with a high mortality rate. We had good result with I.V antibiotic, supportive care and conservative surgical management.

\section{OCCIPITAL ENCEPHALOCELE: REPORT OF CASE SERIES}

doi:10.1136/archdischild-2012-302724.0577

${ }^{1} \mathrm{M}$ Ugras, ${ }^{2} \mathrm{HS}$ Karabekir, ${ }^{3} \mathrm{O}$ Kavak, ${ }^{3} \mathrm{~T}$ S Sen, ${ }^{3} \mathrm{~F}$ Alpay. ${ }^{1}$ Pediatrics, Yeditepe Univ Medical Faculty, Istanbul; '2Neurosurgery; ${ }^{3}$ Pediatrics, Afyon Kocatepe Univ, Afyonkarahisar, Turkey

An encephalocele results from failure of the surface ectoderm to separate from the neuroectoderm. The prevalance ranges from 0.8 to 4 per 10.000 live births. The occiput is the most common site. During a 3 years' period 5 babies with occipital encephalocele were evaluated. All babies were girls. One mother was 45 years old, the others were around 25 years-old. All babies were born with cesarean sectio. Maternal folic acid (FA) consumption revealed that only 2 mothers used FA irregularly, not beginning preconceptionally. The 